\title{
Development of an MeV gamma-ray imaging detector
}

\section{$\operatorname{AUTHOR}(\mathrm{S})$ :}

Takeda, A; Kubo, H; Miuchi, K; Nagayoshi, T; Okada, Y; Orito, R; Takada, A; ... Ueno, M; Bouianov, O; Bouianov, M

\section{CITATION:}

Takeda, A ...[et al]. Development of an MeV gamma-ray imaging detector. IEEE TRANSACTIONS ON NUCLEAR SCIENCE 2004, 51(5): 21402144

ISSUE DATE:

2004-10

URL:

http://hdl.handle.net/2433/49874

\section{RIGHT:}

(c)2004 IEEE. Personal use of this material is permitted. However, permission to reprint/republish this material for advertising or promotional purposes or for creating new collective works for resale or redistribution to servers or lists, or to reuse any copyrighted component of this work in other works must be obtained from the IEEE. 


\title{
Development of an MeV Gamma-Ray Imaging Detector
}

\author{
A. Takeda, H. Kubo, K. Miuchi, T. Nagayoshi, Y. Okada, R. Orito, A. Takada, T. Tanimori, M. Ueno, O. Bouianov, \\ and M. Bouianov
}

\begin{abstract}
An MeV gamma-ray imaging detector which makes a reconstruction of single photons possible has been developed. This $\mathrm{MeV}$ gamma-ray detector is a hybrid detector of a micro time projection chamber (micro-TPC) with a gaseous micro pixel chamber $(\mu$-PIC) readout and an enclosing scintillation camera. We developed a prototype of the MeV gamma-ray imaging detector with the micro-TPC of $10 \times 10 \times 8 \mathrm{~cm}^{3}$ volume and an NaI(Tl) scintillator of $10 \times 10 \times 2.5 \mathrm{~cm}^{3}$ size read by 25 single anode PMTs of $3 / 4^{\prime \prime}$ diameters, and succeeded in reconstructing the gamma-ray events with a radioactive source. The angular resolutions of the prototype detector were limited mainly by the tracking accuracy of the micro-TPC for minimum ionizing particles (MIPs) which is determined by the gas gain of the $\mu$-PIC. We have, thus, developed an electrode structure of the $\mu$-PIC using a new manufacture technology in order to improve the gas gain. As a result, a gas gain which is three-times higher than that of the previous $\mu$-PIC has been obtained. This result is in good agreement with the simulation. Owing to this improvement of the electrode structure, not only the high gas gain but also a good gain uniformity has been achieved.
\end{abstract}

Index Terms-Gamma-Ray Imaging, gaseous detector, micropattern detector, time projection chamber.

\section{INTRODUCTION}

A new imaging detector for reconstructing incident $\mathrm{MeV}$ gamma-rays event by event has been desired for years in astronomy. Since the present gamma-ray detectors with a double or multiple Compton method [1], [2] do not measure the direction of the recoil electron in the Compton scattering process, the direction of the incident gamma-ray is determined only by a Compton event circle. We proposed a new $\mathrm{MeV}$ gamma-ray imaging detector that is a hybrid detector of a micro time projection chamber (micro-TPC) with a gaseous micro pixel chamber $(\mu$-PIC) readout and an enclosing scintillation camera. Since the micro-TPC can detect the three-dimensional fine $(\sim$ sub-millimeter) tracks of the recoil electrons

Manuscript received November 15, 2003; revised April 28, 2004. This work is supported by a Grant-in-Aid for the 21st Century COE "Center for Diversity and Universality in Physics" and a Grant-in-Aid in Scientific Research of the Japan Ministry of Education, Culture, Science, Sports and Technology, and "Ground Research Announcement for Space Utilization" promoted by Japan Space Forum.

A. Takeda, H. Kubo, K. Miuchi, T. Nagayoshi, Y. Okada, R. Orito, A. Takada, T. Tanimori and M. Ueno are with the Department of Physics, Graduate School of Science, Kyoto University, Kyoto 606-8502, Japan.

O. Bouianov is with the Espoo-Vantaa Institute of Technology, Espoo 02101, Finland.

M. Bouianov is with the CSC-Scientific Computing Ltd., Espoo 02650, Finland.

Digital Object Identifier 10.1109/TNS.2004.836154 by electrodes with a pitch of $400 \mu \mathrm{m}$, event by event full reconstructions can be realized using both information from the micro-TPC and the enclosing scintillation camera for measuring scattered gamma-rays. Furthermore, the measured track of the recoil electron gives us an additional redundancy that can be used to reject almost all of the background by the kinematical constraint. A simulation study showed that a $30-\mathrm{cm}$ cubic detector filled with $\mathrm{Xe}$ gas at 1.5 atm has detection efficiencies of $\sim 1 \%$ at $200 \mathrm{keV}$ and $\sim 0.1 \%$ at $1.6 \mathrm{MeV}$ [3].

We developed a prototype of the $\mathrm{MeV}$ gamma-ray imaging detector with the micro-TPC and the $\mathrm{NaI}(\mathrm{Tl})$ scintillator, and succeeded in obtaining the first gamma-ray image by full reconstruction with a radioactive source [3], [4], although angular resolutions and detection efficiencies were not sufficient. The angular resolutions of the prototype detector are limited mainly by the tracking accuracy of the micro-TPC for minimum ionizing particles (MIPs). According to a simulation [5], the gas gain of the $\mu$-PIC with a new electrode structure is expected to be three-times higher than that of the previous $\mu$-PICs, and then the recoil electron tracks are expected to be more precise.

We have, thus, developed the electrode structure of the $\mu$-PIC readout using a new manufacture technology in order to improve the gas gain. The properties of the $\mu$-PIC with the new electrodes have been studied, and a three-times higher gas gain than that of the previous $\mu$-PIC has been obtained, which is in good agreement with the simulation. Moreover, a good gain uniformity and spatial resolution have been achieved.

In this paper, the performance of the $\mu$-PIC with the new electrode structure applied to the $\mathrm{MeV}$ gamma-ray imaging detector is described.

\section{MeV Gamma-RAy IMAging Detector}

\section{A. Concept of Gamma-Ray Reconstruction}

Fig. 1 shows a conceptual structure of the MeV gamma-ray imaging detector with electron tracking in the Compton scattering process and the principle of the incident gamma-ray reconstruction. A micro-TPC with the $\mu$-PIC readout is enclosed by a position sensitive scintillation camera.

If Compton scattering occurs in the gases, both the three-dimensional track $(\vec{e})$ and the energy of the recoil electron $\left(E_{e}\right)$ are measured by the micro-TPC, while the position $\left(\overrightarrow{\gamma^{\prime}}\right)$ and energy of the scattered gamma-ray $\left(E_{\gamma^{\prime}}\right)$ are measured by the scintillation camera. The start and end points of the recoil electron track are distinguished by the difference in the energy deposit. The scattering angle of the gamma-ray $(\phi)$, defined as the angle 


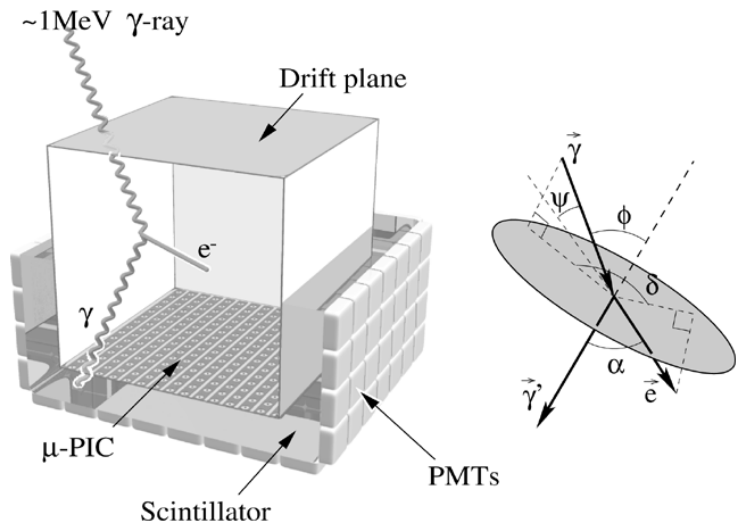

Fig. 1. Conceptual structure of the MeV gamma-ray imaging detector with electron tracking (left), and definitions of the axes (right).

between the incident and scattered gamma-ray $\left(\vec{\gamma}\right.$ and $\left.\overrightarrow{\gamma^{\prime}}\right)$, is calculated by the following equations:

$$
\begin{aligned}
\cos \phi & =1-m_{e} c^{2}\left(\frac{1}{E_{\gamma^{\prime}}}-\frac{1}{E_{\gamma}}\right), \\
E_{\gamma} & =E_{\gamma^{\prime}}+E_{e}
\end{aligned}
$$

where $E_{\gamma}$ is the energy of the incident gamma-ray, and $m_{e}$ is the mass of the electron. Since we can determine not only the angle $\phi$, but also another angle $\delta$ between the projected vectors of the recoil electron and the incident gamma-ray on the normal plane to the scattered gamma-ray, a full reconstruction of the incident gamma-ray can be realized. We also have a redundant measured value $\alpha$ that can be used for background rejection (hereafter called " $\alpha$-cut") from the consistency of the measured $\alpha$ and the calculated one such as,

$$
\cos \alpha=\left(1-\frac{m_{e} c^{2}}{E_{\gamma^{\prime}}}\right) \sqrt{\frac{E_{e}}{E_{e}+2 m_{e} c^{2}}} .
$$

Since no collimators are necessary, a large field of view can be realized.

\section{B. Prototype of the MeV Gamma-Ray Imaging Detector}

We developed a prototype of the $\mathrm{MeV}$ gamma-ray imaging detector with a micro-TPC of $10 \times 10 \times 8 \mathrm{~cm}^{3}$ volume and an $\mathrm{NaI}(\mathrm{Tl})$ scintillator of $10 \times 10 \times 2.5 \mathrm{~cm}^{3}$ size read by 25 single anode PMTs of $3 / 4^{\prime \prime}$ diameters as shown in Fig. 2.

The micro-TPC consists of a $10 \times 10 \mathrm{~cm}^{2} \mu$-PIC and a drift length of $8 \mathrm{~cm}$. The $\mu$-PIC is a gaseous two-dimensional position-sensitive detector manufactured by printed circuit board (PCB) technology [6], [7]. With PCB technology, large area detectors could be mass-produced cheaply. A schematic structure of the $\mu$-PIC is shown in Fig. 3. Anode and cathode strips are formed orthogonally on both sides of a $100 \mu \mathrm{m}$ thick polyimide substrate with a pitch of $400 \mu \mathrm{m}$. The cathode strip has circular holes with a diameter of $260 \mu \mathrm{m}$. The anode pillars are grown on the anode strip through the substrate at the center of each cathode hole using electrochemical metal deposition. A gas avalanche occurs around the anode pillar due to a strong electric field. On the other hand, the electric field is weaker at the edge of the cathode because of its longer circumference. This structure should provide a higher gas gain than a microstrip gas

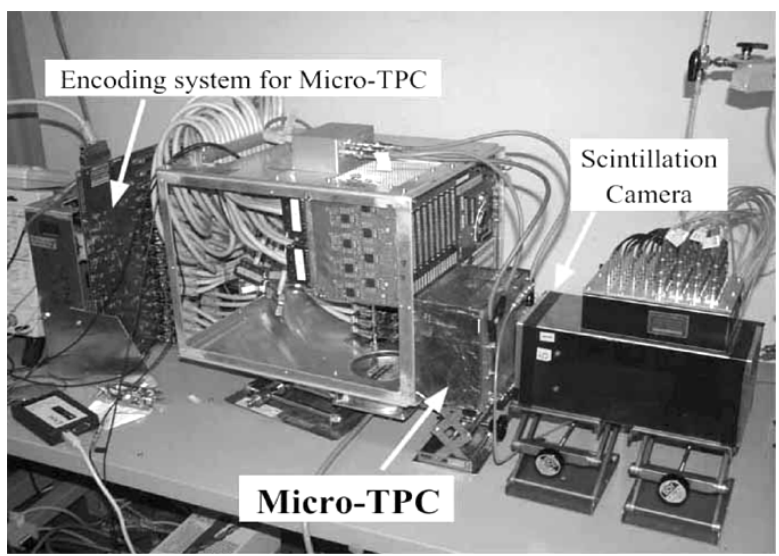

Fig. 2. Prototype of the gamma-ray imaging detector.

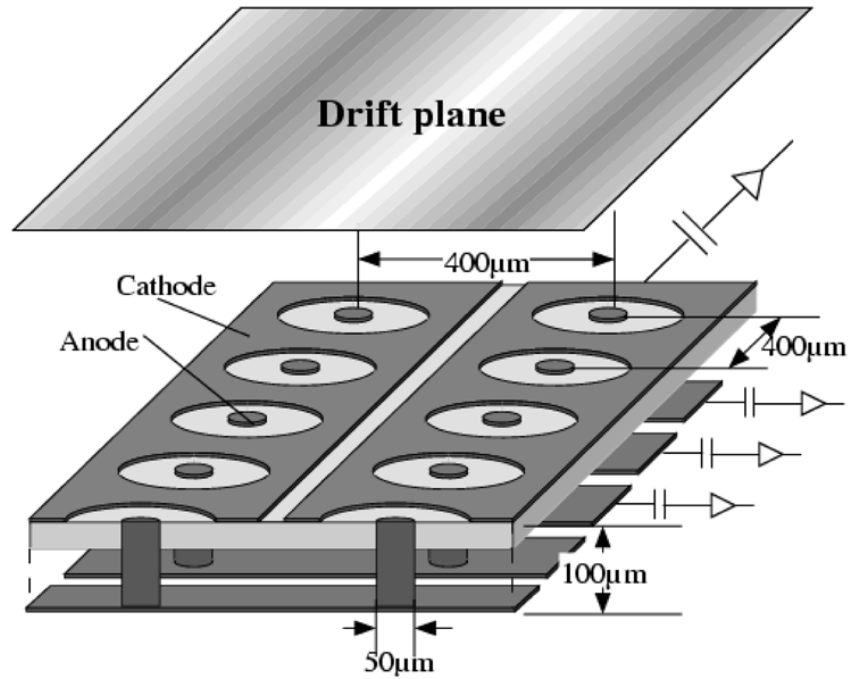

Fig. 3. Schematic structure of the $\mu$-PIC.

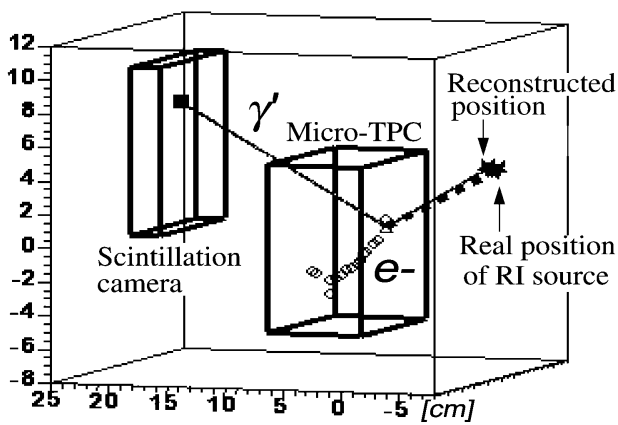

Fig. 4. Typical gamma-ray event from ${ }^{137} \mathrm{Cs}$ reconstructed correctly. The filled star shows the real position of the RI source. The Compton scattered gamma-ray detected by the scintillation camera is shown by the square symbol. The electron track detected by the micro-TPC is shown by the open circles. The reconstructed position where the radioactive source is set is shown by the gray star.

chamber (MSGC [8], [9]) without any serious damages that can occur in an MSGC in the case of a discharge event. We have already examined the performance of several $\mu$-PICs with various structures of electrodes [10]. In a previous performance test, a maximum gas gain of $1.5 \times 10^{4}$ and a long term stability over 1000 hours at a gas gain of 5000 were achieved for an $\mathrm{Ar}-\mathrm{C}_{2} \mathrm{H}_{6}$ (80:20) gas mixture [11] without any other intermediate gas multipliers, such as Gas Electron Multipliers (GEMs) 

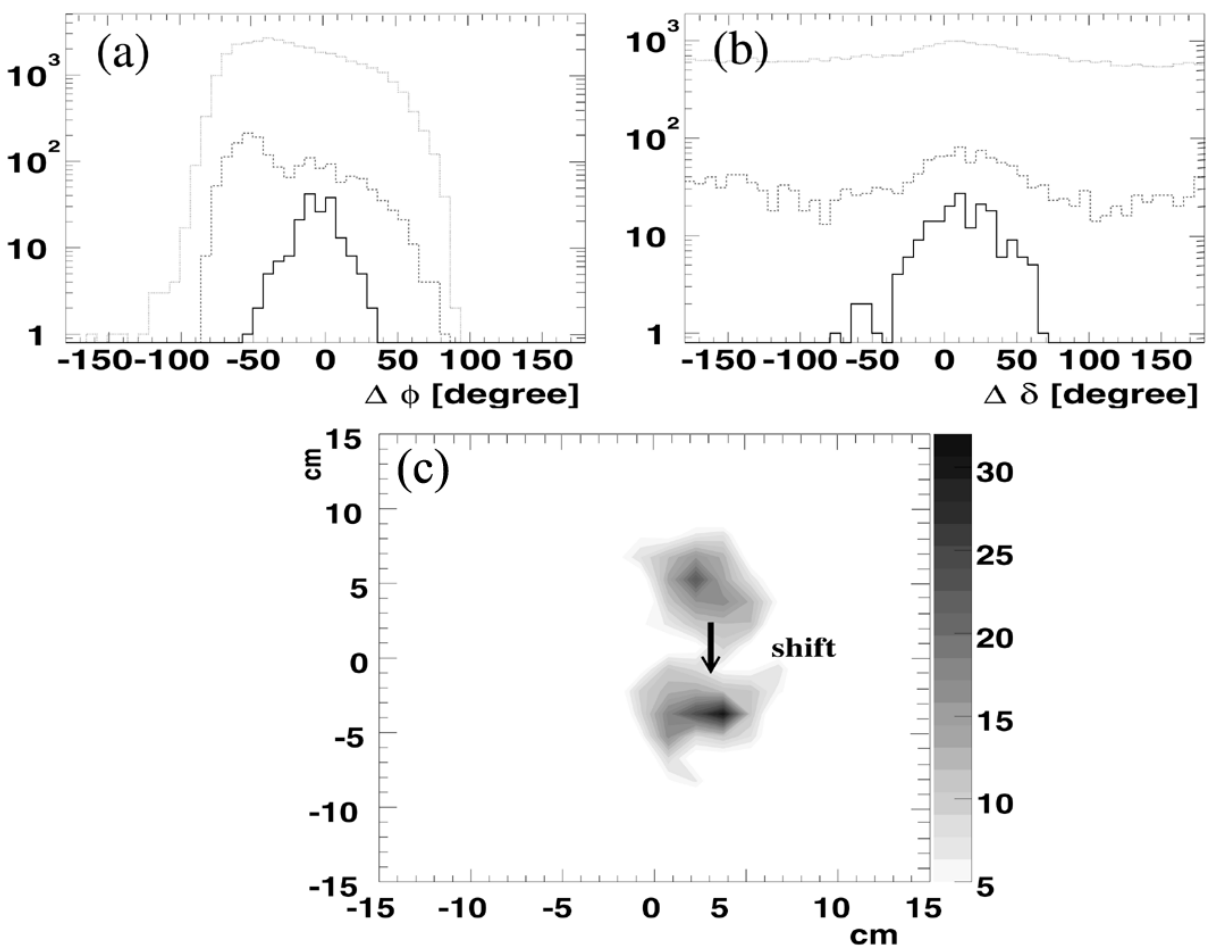

Fig. 5. Angular resolutions of two angles for gamma-ray reconstruction, $\phi$ (a) and $\delta$ (b). The dotted-lines, dashed-lines, and solid-ones show the raw data, after applying cuts of the fiducial volume and tracks with the small number of hits, and also after $\alpha$-cuts, respectively. Two reconstructed images were plotted (c), where a shift of the source position (40 degrees) was obviously seen.

[12]. This simple structure of the $\mu$-PIC is expected to allow a stable operation for detecting high $d E / d x$ particles to be possible. A data acquisition (DAQ) system for the $\mu$-PIC consisting of amplifier-shaper-discriminator (ASD) cards, a position encoding module and a VME memory module was developed [13]. The ASD cards based on the ASD IC for the Thin Gap Chamber (TGC-ASD) in the LHC ATLAS Experiment have been developed so that the integration constant is suitable for the $\mu$-PIC [14]. Signals from the $\mu$-PIC are amplified and discriminated by the ASD cards. Discriminated signals (LVDS-level pulse) are encoded by the position encoding module consisting of five field programmable gate arrays (FPGAs), and are recorded by the memory module with an internal clock of $20 \mathrm{MHz}$. Analog signals from the 16 cathode strips are summed and digitized by the $100 \mathrm{MHz}$ flash ADC (FADC: REPIC RPV-160) to determine the event energy.

The scintillation camera for detecting the scattered gammarays consists of a $10 \times 10 \times 2.5 \mathrm{~cm}^{3} \mathrm{NaI}(\mathrm{Tl})$ plate and an array of $5 \times 53 / 4^{\prime \prime}$ Hamamatsu R1166 PMTs. We are now planning to improve the scintillation camera covering only the bottom of the micro-TPC (Fig. 2) so that four sides will be covered with it. Each signal from the PMT is recorded by a peak hold ADC (PHADC) after pulse shaping, and the center of gravity of the position is interpolated from these pulse heights. The energy and position resolutions (FWHM) of the scintillation camera for 662 $\mathrm{keV}$ gamma-rays are $9 \%$ and $7.5 \mathrm{~mm}$, respectively. When hit signals from the micro-TPC exist within $2 \mu$ s after the trigger of the scintillation camera, data from both the micro-TPC and the scintillation camera are recorded.

We studied the imaging performance of the prototype $\mathrm{MeV}$ gamma-ray detector with a radio isotope (RI) source of ${ }^{137} \mathrm{Cs}$
(662 keV). The micro-TPC was filled with $\mathrm{Ar}-\mathrm{C}_{2} \mathrm{H}_{6}$ (80:20) gas at $1 \mathrm{~atm}$. The RI source was placed $5 \mathrm{~cm}$ away from the opposite side of the micro-TPC, which faced the scintillation camera in order to effectively collect the forward scattering events. Recoil electron tracks were reconstructed by connecting hit points obtained from the $\mu$-PIC readout in order of the recording time. Fig. 4 shows one of the well-reconstructed events taken by the prototype $\mathrm{MeV}$ gamma-ray detector. In an off-line analysis, we required the hit positions of both fiducial volumes of the micro-TPC, the minimum number of hit positions of the track in the micro-TPC (at least three points per track), and the scintillation camera, and the kinematical constraint of the $\alpha$ angle. Since the energy calibration of the micro-TPC was not sufficiently accurate to sum with the energy of the scintillation camera, the initial energy of gamma-rays $(662 \mathrm{keV})$ was treated as a known parameter. Fig. 5 shows the distributions of two angles of $\phi$ and $\delta$ obtained by event reconstructions. Almost all of the background events with $|\Delta \phi|>50^{\circ}$ and $|\Delta \delta|>70^{\circ}$ were rejected by the $\alpha$-cuts as shown in Fig. 5. The resultant angular resolutions of $\phi$ and $\delta$ were 20 and 25 degrees at RMS, respectively [4]. When we moved the source position by 40 degrees, the position of the reconstructed gamma-ray image moved correctly (Fig. 5). The reconstructing of the incident gamm-ray has been confirmed, although the angular resolutions were still not sufficient.

The angular resolutions of this prototype $\mathrm{MeV}$ gamma-ray detector were limited mainly by the tracking accuracy of the micro-TPC for MIPs, which is determined by the gas gain of the $\mu$-PIC. In order to improve the gas gain, we have developed the electrode structure of the $\mu$-PIC using the new manufacture technology. 
(a) Electroless plating

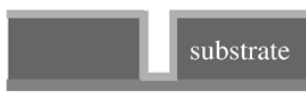

(b) Via-fill plating

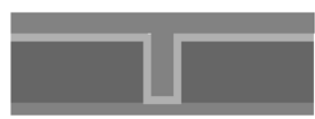

(c) Surface etching

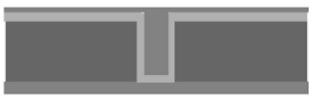

(d) Electrode etching

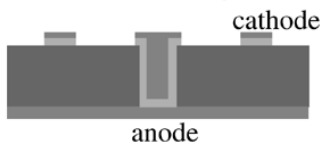

Fig. 6. Manufacture process of the new $\mu$-PIC. First, the whole surface of the substrate is covered with a copper layer by electroless plating (a), and via-fill plating (b). Second, the whole surface of the substrate is etched until its thickness is reduced to $\sim 10 \mu \mathrm{m}$ (c). Last, anode and cathode electrodes are etched together (d).

\section{III. $\mu$-PIC With THE NeW EleCtrodes}

\section{A. Optimization of the Electrodes}

An optimum electrode structure was investigated using Maxwell 3D Field Simulator [15] and Garfield [16]. According to a simulation, the previous $\mu$-PIC, whose anode pillars end $20 \mu \mathrm{m}$ below the surface of the substrate, suffers from gas gain degradation because a large fraction of electron cloud due to incident particles does not reach the anodes [17]. The collection efficiency of the electrons is estimated to be only $30 \%$. On the contrary, an efficiency of $97 \%$ is expected to be achieved if the tops of the anodes are $10 \mu \mathrm{m}$ higher than the surface of the substrate [5].

Since manufacturing this type of anode electrode by the conventional technology has certain difficulties, a new technology is used. In this new technology, the anode and cathode electrodes are etched after the whole substrate surface is covered with a copper layer by electroless plating and via-fill plating as shown in Fig. 6.

A new $\mu$-PIC with a detection area of $10 \times 10 \mathrm{~cm}^{2}$ has been manufactured using this new technology. The development of this new type of $\mu$-PIC with a detection area of $30 \times 30 \mathrm{~cm}^{2}$ is also now in progress.

\section{B. Performance of the New $\mu$-PIC}

We irradiated with a noncollimated radioactive source of ${ }^{55} \mathrm{Fe}$ and measured the output charge from $32 \times 256$ pixels of the new $\mu$-PIC in an Ar- $\mathrm{C}_{2} \mathrm{H}_{6}$ (80:20) gas mixture of atmospheric pressure. From the obtained values of the charges, the gas gains of the new $\mu$-PIC are calculated and plotted as a function of the anode voltage in Fig. 7. As a result, a three-times higher gas gain than that of the previous $\mu$-PIC was achieved. This result is in good consistency with the estimation of the 3D simulation. In Fig. 8, the gain map in which each divided region represents the gas gain obtained from $32 \times 32$ pixels is shown. The gas gain in the whole detection area was uniform within $7 \%(\sigma)$. The gain uniformity was significantly improved compared with that of the previous $\mu$-PIC.

In Fig. 9, the energy spectrum obtained from the whole detection area $\left(10 \times 10 \mathrm{~cm}^{2}\right)$ by irradiating the X-ray source of ${ }^{55} \mathrm{Fe}$ is shown. The energy resolution is $30 \%$ (FWHM) for the $5.9 \mathrm{keV}$ X-rays.

We took the X-ray image of the test chart with the X-ray generator (Kevex X-Ray CU028, tungsten target). The acceleration

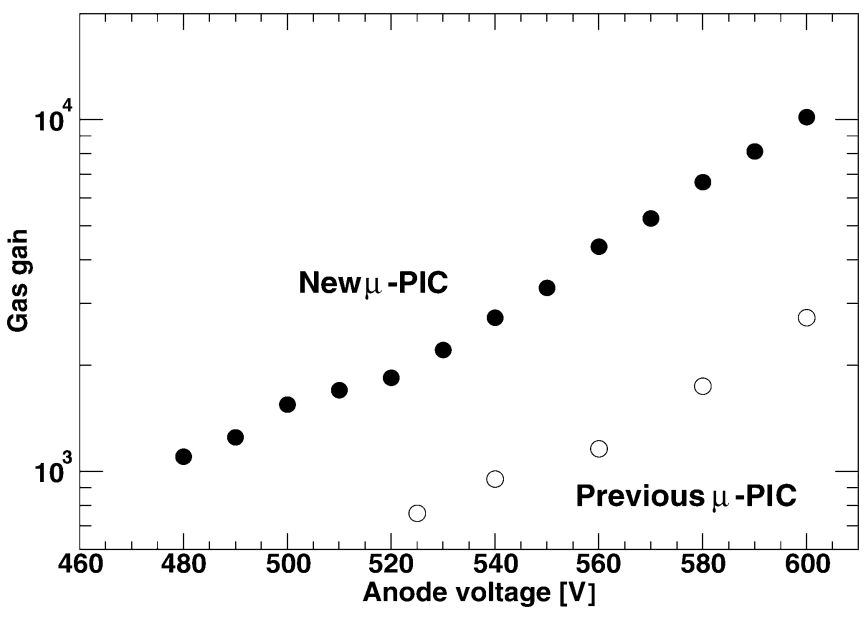

Fig. 7. Gas gains as a function of the anode voltage of which the $\mu$-PIC with the new and previous electrodes are shown by closed circles and open circles, respectively.

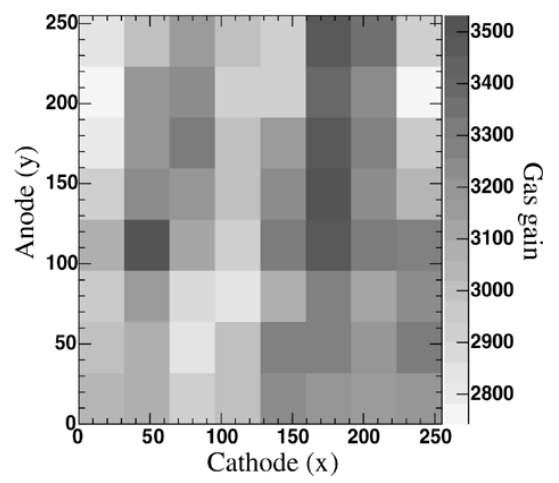

Fig. 8. Obtained gain map of the $\mu$-PIC with the new electrodes.

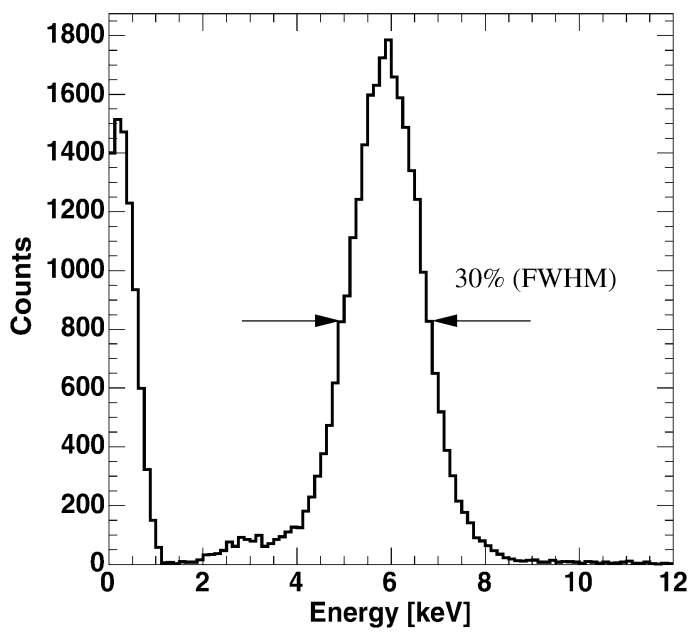

Fig. 9. Energy spectrum obtained from the whole detection area $(10 \times 10$ $\mathrm{cm}^{2}$ ) by irradiating the $\mathrm{X}$-ray source of ${ }^{55} \mathrm{Fe}$.

voltage of the X-ray generator was $12 \mathrm{kV}$ with $1 \mathrm{~mm}$ thick aluminum filtering low energy $\mathrm{X}$-rays. In order to achieve clear images, we used gas mixture of $\mathrm{Xe}^{-} \mathrm{C}_{2} \mathrm{H}_{6}$ (70:30) at $1 \mathrm{~atm}$. The practical range of the $12 \mathrm{keV}$ electron in argon gas is $1 \mathrm{~mm}$, while that in xenon gas is $0.2 \mathrm{~mm}$. The detection depth was set to be $2 \mathrm{~mm}$. The test chart is $5 \times 5 \mathrm{~cm}^{2}$ area, and slits of various widths are scribed in the $0.05 \mathrm{~mm}$ thick lead layer on the plastic 


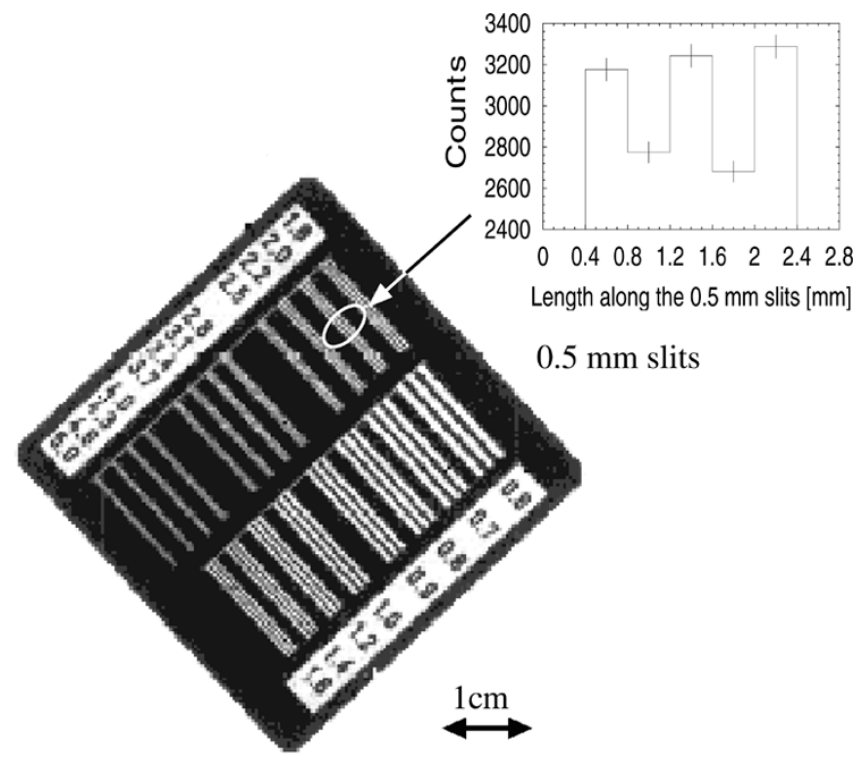

Fig. 10. X-ray image of the test chart and the projected image along the 0.5 $\mathrm{mm}$ slits.

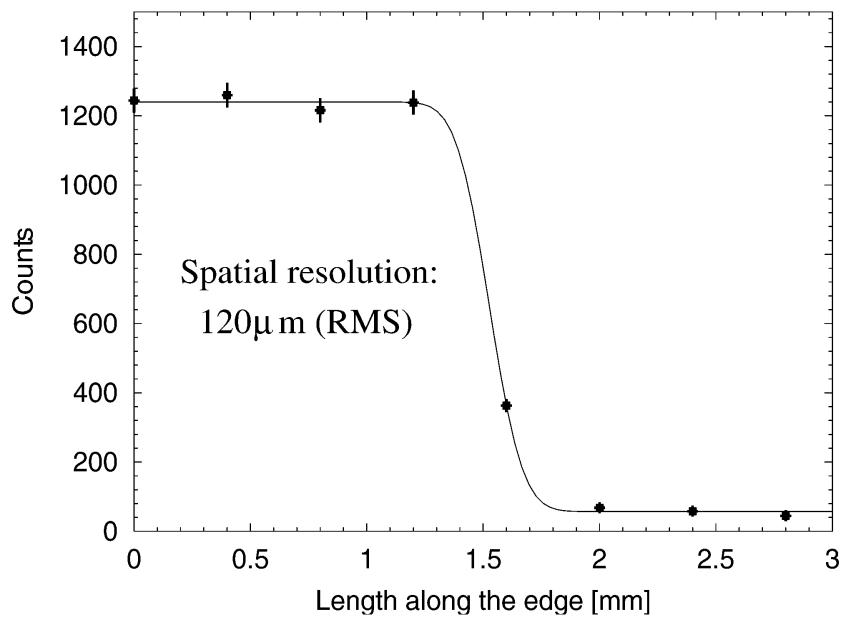

Fig. 11. Projected image of the test chart edge and the best fit function of $a_{1}+a_{2} \cdot \operatorname{erf}\left(\left(x-a_{3}\right) / \sqrt{2} a_{4}\right)$, where $\operatorname{erf}(x)=1 / \sqrt{\pi} \cdot \int_{0}^{x} \exp \left(-t^{2}\right) d t$ is the error function and $a_{1}, a_{2}, a_{3}$ and $a_{4}$ are the fitting parameters.

plate. Fig. 10 shows the obtained X-ray image and the projected image along the $0.5 \mathrm{~mm}$ slits. The slits of $0.5 \mathrm{~mm}$ width are clearly separated.

We measured the spatial resolution by the knife edge method. In order to reduce the range of the electrons, the acceleration voltage of the X-ray generator was set at $10 \mathrm{kV}$ for this measurement. The active area was set to be $8 \times 8 \mathrm{~cm}^{2}$, and the test chart image was projected along one of the edges for $2 \mathrm{~cm}$. Fig. 11 shows the profile of the test chart edge and the best fit function of $a_{1}+a_{2} \cdot \operatorname{erf}\left(\left(x-a_{3}\right) / \sqrt{2} a_{4}\right)$, where $\operatorname{erf}(x)=$ $1 / \sqrt{\pi} \cdot \int_{0}^{x} \exp \left(-t^{2}\right) d t$ is the error function and $a_{1}, a_{2}, a_{3}$ and $a_{4}$ are the fitting parameters. The calculated spatial resolution $\left(a_{4}\right)$ was $120 \mu \mathrm{m}$ (RMS). This result is close to the theoretical limit of the spatial resolution expected as the formula, $\sigma=d / \sqrt{12}=115 \mu \mathrm{m}$, where $d=400 \mu \mathrm{m}$ is the electrode pitch.

\section{CONCLUSION}

We have developed the $10 \times 10 \mathrm{~cm}^{2} \mu$-PIC with the new electrode structure using the new manufacture technology. A three-times higher gas gain than that of the previous $\mu$-PIC, which is enough to detect MIPs for the MeV gamma-ray imaging detector and a good gain uniformity of $7 \%$ (RMS) has been achieved. The energy resolution obtained from the whole detection area $\left(10 \times 10 \mathrm{~cm}^{2}\right)$ was $30 \%(\mathrm{FWHM})$ for the 5.9 $\mathrm{keV} \mathrm{X}$-rays. As an X-ray imaging detector, a good spatial resolution of $120 \mu \mathrm{m}$ (RMS), which is close to the theoretical limit with $400 \mu \mathrm{m}$ pitch electrodes has been obtained. A large area $\left(30 \times 30 \mathrm{~cm}^{2}\right) \mu$-PIC with this new type of electrode is now being manufactured. We are also developing a new scintillation camera enclosing five surfaces of the micro-TPC. From these improvements and a suitable integration constant of ASD cards [14], the angular resolutions of the $\mathrm{MeV}$ gamma-ray imaging detector are expected to be less than 10 degrees above $500 \mathrm{keV}$.

\section{REFERENCES}

[1] V. Shönfelder, "The imaging gamma-ray telescope COMPTEL aboard GRO," Adv. Space Res., vol. 11, no. 8, pp. 313-322, 1991.

[2] T. Kamae, R. Enomoto, and N. Hanada, "A new method to measure energy, direction, and polarization of gamma rays," Nucl. Instrum. Methods, vol. A260, no. 1, pp. 254-257, Oct. 1987.

[3] R. Orito, H. Kubo, K. Miuchi, T. Nagayoshi, A. Ochi, A. Takada, T. Tanimori, and $\mathrm{M}$. Ueno, "A novel design of the $\mathrm{MeV}$ gamma-ray imaging detector with Micro-TPC," Nucl. Instrum. Methods, vol. A513, no. 1-2, pp. 408-412, Nov. 2003.

[4] R. Orito, H. Kubo, K. Miuchi, T. Nagayoshi, A. Takada, A. Takeda, T. Tanimori, and M. Ueno, "Compton gamma-ray imaging detector with electron tracking," Nucl. Instrum. Methods, vol. A525, no. 1-2, pp. 107-113, Jun. 2004

[5] T. Nagayoshi, H. Kubo, K. Miuchi, R. Orito, A. Takada, A. Takeda, T. Tanimori, and M. Ueno, "Development of $\mu$-PIC and its imaging properties," Nucl. Instrum. Methods, vol. A525, no. 1-2, pp. 20-27, Jun. 2004.

[6] A. Ochi, T. Nagayoshi, S. Koishi, T. Tanimori, T. Nagae, and M. Nakamura, "A new design of the gaseous imaging detector: Micro pixel chamber," Nucl. Instrum. Methods, vol. A471, no. 1-2, pp. 264-267, Sept. 2001.

[7] _ , "Development of Micro pixel chamber," Nucl. Instrum. Methods, vol. A478, no. 1-2, pp. 196-199, Feb. 2002.

[8] A. Oed, "Position-sensitive detector with microstrip anode for electron multiplication with gases," Nucl. Instrum. Methods, vol. A263, no. 2-3, pp. 351-359, Jan. 1988

[9] T. Tanimori, A. Ochi, S. Minami, and T. Nagae, "Development of an imaging microstrip gas chamber with a $5 \times 5 \mathrm{~cm}^{2}$ area based on multichip module technology," Nucl. Instrum. Methods, vol. A381, no. 2-3, pp. 280-288, Nov. 1996.

[10] T. Nagayoshi, H. Kubo, K. Miuchi, A. Ochi, R. Orito, A. Takada, T. Tanimori, and M. Ueno, "Performance of large area Micro pixel chamber," Nucl. Instrum. Methods, vol. A513, no. 1-2, pp. 277-281, Nov. 2003.

[11] K. Miuchi, H. Kubo, T. Nagayoshi, A. Ochi, R. Orito, A. Takada, T. Tanimori, and M. Ueno, "Performance of the TPC with Micro pixel chamber readout: Micro-TPC," IEEE Trans. Nucl. Sci., vol. 50, pp. 825-830, Aug. 2003.

[12] F. Sauli, "GEM: A new concept for electron amplification in gas detectors," Nucl. Instrum. Methods, vol. A386, pp. 531-534, Feb. 1997.

[13] H. Kubo, K. Miuchi, T. Nagayoshi, A. Ochi, R. Orito, A. Takada, T. Tanimori, and M. Ueno, "Development of the time projection chamber with Micro pixel electrodes," Nucl. Instrum. Methods, vol. A513, no. 1-2, pp. 94-98, Nov. 2003.

[14] R. Orito, O. Sasaki, H. Kubo, K. Miuchi, T. Nagayoshi, A. Takada, A. Takeda, T. Tanimori, and M. Ueno, , in these proceedings.

[15] Maxwell 3D Field Simulator. Ansoft Corp.. [Online]. Available: http://www.ansoft.com

[16] R. Veenhof, "Garfield, recent developments," Nucl. Instrum. Methods, vol. A419, no. 2-3, pp. 726-730, Dec. 1998.

[17] T. Nagayoshi, "Development of MicroPixel Chamber and Systematic Study on the Electrode Structure," Ph.D. dissertation, Kyoto University, 2004. 